\title{
Superfamilia Scarabaeoidea (Insecta: Coleoptera) como elemento bioindicador de perturbación antropogénica en un parque nacional amazónico
}

\author{
Samuel Eduardo Otavo ${ }^{1}$, Ángela Parrado-Rosselli ${ }^{1}$ \& Jorge Ari Noriega ${ }^{2,3}$ \\ 1. Grupo de Investigación Uso y Conservación de la Diversidad Forestal, Facultad del Medio Ambiente y Recursos \\ Naturales Universidad Distrital Francisco José de Caldas, Bogotá, Colombia; geniusforestal@gmail.com, \\ aparrador@udistrital.edu.co \\ 2. Laboratorio de Zoología y Ecología Acuática - LAZOEA, Universidad de Los Andes, Bogotá, Colombia; \\ jnorieg@hotmail.com \\ 3. Scarabaeinae Research Network - ScarabNet
}

Recibido 16-IV-2012. Corregido 18-IX-2012. Aceptado 12-X-2012.

\begin{abstract}
Scarabaeoidea superfamily (Insecta: Coleoptera) as a bioindicator element of anthropogenic disturbance in an amazon national park. Insects have been recognized to be important indicators of the quality elements of ecosystems, among others, because of their rapid response to environmental variability and ease cost-effective capture. In this work we evaluated whether beetles of the Scarabaeoidea superfamily may be used as bioindicators of anthropogenic disturbance of Amazonian terra firme rain forests, in order to provide guidelines for monitoring strategies of the Amacayacu National Park. We considered three different levels of anthropogenic disturbance (i.e. low, medium, high) in 12 transects (four in each intervention level), and caught all beetle species of this superfamily. Three interception traps, two light traps, three pitfalls and four bottle fruit traps were used per transect, as well as manual catch. In total, 593 individuals belonging to 92 species, 44 genera and seven families were collected. Scarabaeidae ( $n=232,27 \mathrm{spp}$.) and Dynastidae ( $\mathrm{n}=161,26 \mathrm{spp}$.) were the families with the highest number of individuals and species, while Aphodiidae, Cetoniidae and Geotrupidae exhibited the lowest. The most abundant species per family were Ateuchus sp. (33.2\%) from Scarabaeidae, Cyclocephala verticalis (55.9\%) from Dynastidae, Astaena sp. (75.8\%) from Melolonthidae, Ceratocanthus amazonicus (66.7\%) from Ceratocanthidae y Chaetodus asuai (96.8\%) from Hybosoridae. Results showed that the number of species and individuals increased with the anthropogenic disturbance. The Margalef and Shannon indexes also revealed that the highest richness and equity occurred in the high-disturbed site, respectively. Dynastidae exhibited the highest number of exclusive species per gradient, while Scarabaeidae shared most of its species. Ten species were recorded in the three disturbance levels, 26 species in two and 56 species were exclusive to one level. The most abundant species Chaetodus asuai (Hybosoridae) presented a significant decrease in the number of individuals as disturbance increased, while an inverse relationship occurred for C. verticalis (Dynastidae). For Astaena sp. (Melolonthidae) and Ligyrus gyas (Dynastidae) a high number of individuals was recorded and were exclusive for one forest site (low and high disturbed forest, respectively). The complete superfamily cannot be used like a single bioindicator element because it displays, in a specific level, very different behaviors in each disturbance condition. We discuss the use of richness and diversity as indicators of disturbance, while we highlight abundance of particular taxa as a better metric for disturbance. Rev. Biol. Trop. 61 (2): 735-752. Epub 2013 June 01.
\end{abstract}

Key words: Amazon forest, beetles, Dynastidae, ecological integrity, Hybosoridae, indicators, Scarabaeidae.

Una de las herramientas más importantes para la evaluación del estado de conservación de las áreas protegidas, que contribuye a la toma de decisiones de manejo, es el análisis de la integridad ecológica. En Colombia, el
Sistema de Parques Nacionales Naturales ha desarrollado una metodología para evaluar la integridad ecológica en sus áreas, la cual ha definido una serie de indicadores paisajísticos y poblacionales según características de 
pertinencia, credibilidad, factibilidad y claridad (Zambrano et al. 2007). No obstante, una de las limitantes fundamentales a la hora de aplicar dicha metodología es la falta de claridad acerca de los elementos de la biodiversidad sobre los cuales aplicar los indicadores poblacionales y cuales resultados son realmente un reflejo del estado de conservación de las áreas protegidas y del grado de perturbación, para de esta manera poder tomar decisiones informadas de forma rápida y eficaz (Fernández 2003, Niemi \& McDonald 2004, Caro et al. 2005).

Los indicadores utilizados en integridad ecológica deben ser variables medibles que tienen como objetivo examinar y monitorear cambios en la composición, estructura y función de los sistemas ecológicos ante el impacto de las perturbaciones ambientales (Stork et al. 1997, Noss 1999, Dale \& Beyeler 2001, Carignan \& Villard 2002). A nivel de especies y poblaciones, los indicadores más utilizados son la riqueza, la abundancia, la distribución espacial y estructura (Magurran 1988, Hodkinson \& Jackson 2005). Los elementos de la biodiversidad sobre los cuales se pueden aplicar estos indicadores pueden ser especies, gremios o comunidades, que sean representativos de la estructura y funcionalidad del ecosistema y que permitan medir cambios a lo largo del tiempo dada su sensibilidad a presiones o perturbaciones naturales y/o humanas (Noss 1990, 1999, Kremen 1992, Carignan \& Villard 2002, Caro et al. 2005).

En este contexto, diferentes grupos de insectos han sido reconocidos por ser importantes elementos bioindicadores de la calidad de los ecosistemas, al poseer características tales como una alta diversidad, importancia funcional, fidelidad ecológica, asociación estrecha con otras especies, rápida respuesta a la variabilidad ambiental y facilidad de captura costoefectiva (Krebs 1988, Halffter \& Favila 1993, Morón 1997, Noss 1999, Celi \& Dávalos 2001, García \& Pardo 2004, Pearce \& Venier 2006, Gardner et al. 2008). Además, ha sido demostrada su sensibilidad a los cambios ocasionados por la intervención del ser humano (Escobar \& Chacón 2000, Basset et al. 2004, Pearce \& Venier 2006, Noriega et al. 2007a). De estos, los coleópteros son hoy en día uno de los grupos más reconocidos y utilizados para el monitoreo y establecimiento de áreas protegidas (Morón 1997, Anderson \& Ashe 2000, Schuster et al. 2000, Campos \& Fernández 2002).

A pesar de lo anterior, la mayoría de estudios se han enfocado en las familias Scarabaeidae y Carabidae (Celi \& Dávalos 2001, Vitolo 2004, Nichols et al. 2007, Noriega et al. 2007a) mientras que aproximaciones a otras familias o a varios taxa de manera simultánea son escasas (Morón 1997, Neita et al. 2006, Schuster et al. 2000). Más aun, los pocos estudios que han utilizado estos grupos como bioindicadores en Colombia se han centrado primordialmente en las familias Dynastidae y Melolonthidae (Restrepo et al. 2003, Neita et al. 2006, Suárez \& Amat-García 2007, Pardo-Locarno et al. 2011), Scarabaeidae (Howden \& Nealis 1975, Amat et al. 1997, Escobar 1997, Amézquita et al. 1999, Escobar 2000, Noriega et al. 2007b, 2008, Concha et al. 2010, Martínez et al. 2010, Solís et al. 2011) y Passalidae (Amat et al. 2004, Amat-García \& Reyes-Castillo 2007, JiménezFerbans \& Amat-García 2009).

Teniendo en cuenta la sensibilidad de este grupo ante procesos de perturbación, fragmentación y caza (Nichols et al. 2007, 2008, 2009) y por ende su potencial papel como bioindicador, así como la alta diversidad de la superfamilia Scarabaeoidea en la Amazonía (Campos \& Fernández 2002, Pulido et al. 2003, Restrepo et al. 2003, Amat-García \& Reyes-Castillo 2007, Noriega et al. 2007b, 2008), el objetivo principal de este estudio fue realizar una primera evaluación de si los escarabajos pertenecientes a la superfamilia podrían ser utilizados como elementos indicadores del grado de perturbación antropogénica de los bosques húmedos amazónicos de tierra firme, con el fin de aportar información a las estrategias de monitoreo, planificación y análisis de integridad ecológica del Parque Nacional 
Natural (PNN) Amacayacu y de las áreas protegidas amazónicas.

\section{MATERIALES Y MÉTODOS}

Área de estudio: El trabajo se realizó en el PNN Amacayacu, localizado en la margen izquierda del río Amazonas, en jurisdicción de los municipios de Leticia y Puerto Nariño y el Corregimiento Departamental de Tarapacá, en Colombia (Ruiz et al. 2007) (Fig. 1). Según el sistema de clasificación por zonas de vida propuesto por Holdridge et al. (1971) el parque es un bosque húmedo tropical y presenta un clima húmedo tropical según la clasificación de Köppen (1936). El régimen de lluvias es unimodal-biestacional con una precipitación media anual de $2836 \mathrm{~mm}$.

El sitio de trabajo se localizó en los bosques de tierra firme de la zona sur del parque. Este tipo de bosque es la unidad dominante tanto de la Amazonía colombiana como del parque (Rudas \& Prieto 2005, Parques Nacionales Naturales 2005, Ruiz et al. 2007). El dosel superior es continuo, fluctuando entre 30 y $45 \mathrm{~m}$, con árboles emergentes que sobrepasan los 50m (Parques Nacionales Naturales 2005). Las familias de plantas más importantes son Fabaceae, Rubiaceae, Melastomataceae, Moraceae, Annonaceae y Arecaceae y las especies dominantes son Eschweilera coriacea, Iriartea deltoidea, Pseudolmedia laevigata, Mauritia flexuosa y Micandras pruceana (Rudas \& Prieto 2005).

Antes del establecimiento del parque en 1975, la zona sur fue utilizada para cultivos artesanales y cultivos ilícitos. En el área se encuentran tres comunidades indígenas: Palmeras, San Martín de Amacayacu y Mocagua, que tienen incidencia directa e indirecta en el área del parque. De tal forma que la región es un mosaico compuesto por bosque maduro primario con diferentes niveles de degradación (extracción selectiva) y bosque secundario en diferentes estadios de sucesión (Gruezmacher $\&$ Duivenvoorden 2008), presentando un completo gradiente de perturbación.
Selección de los sitios de muestreo: Se seleccionaron tres sitios de muestreo de acuerdo al grado de perturbación antropogénica de los bosques: baja, media y alta perturbación. Estos se determinaron con base en la interpretación de material cartográfico, el análisis de documentos relacionados con el uso, manejo y descripción del parque (Pinilla 2004, Parques Nacionales Naturales 2005, Ruiz et al. 2007) y el conocimiento de las comunidades indígenas que tienen incidencia en el bosque (Otavo 2010). Es importante resaltar que la cobertura de los bosques en la zona es continua, por lo que no existe un efecto de fragmentación.

La zona de alta perturbación (BA) está ubicada a una distancia inferior a $7 \mathrm{~km}$ de las comunidades indígenas ( $3^{\circ} 48^{\prime} 30^{\prime}$ ' $\left.\mathrm{S}-70^{\circ} 16^{\prime} 00^{\prime} \mathrm{W}\right)$, caracterizándose por la presencia de bosques secundarios con 30-35 años de regeneración y dosel discontinuo. Es utilizada frecuentemente para turismo, cacería y extracción de productos forestales no maderables. Según las comunidades indígenas, actualmente no existen productos maderables para la extracción ya que en años pasados fueron fuertemente explotados (Otavo 2010).

La zona de media perturbación (BM) está ubicada a una distancia entre 9 y $12 \mathrm{~km}$ de las comunidades indígenas $\left(3^{\circ} 43^{\prime} 00^{\prime \prime} \mathrm{S}\right.$ $\left.70^{\circ} 16^{\prime} 20^{\prime \prime} \mathrm{W}\right)$. Este bosque es menos visitado por habitantes locales y el número de senderos es bajo en comparación a los encontrados en BA. Los pocos senderos son utilizados por cazadores, ecoturismo no frecuente y desplazamiento entre comunidades. Hace 30-50 años la zona estuvo permanentemente ocupada debido a una maloca indígena Ticuna, pero entre 15-20 años, migraron a las riberas del río Amazonas. Actualmente, el uso principal es para extracción ilegal de madera, la cual es estacional ya que la única forma para sacarla es por medio fluvial (época de lluvias). Eventualmente se realizan faenas de recolección de productos no maderables como frutos, semillas, resinas, cortezas, raíces, hojas y tallos que son utilizados en la fabricación de tintes, artesanías, alimento o medicinas (Otavo 2010). 


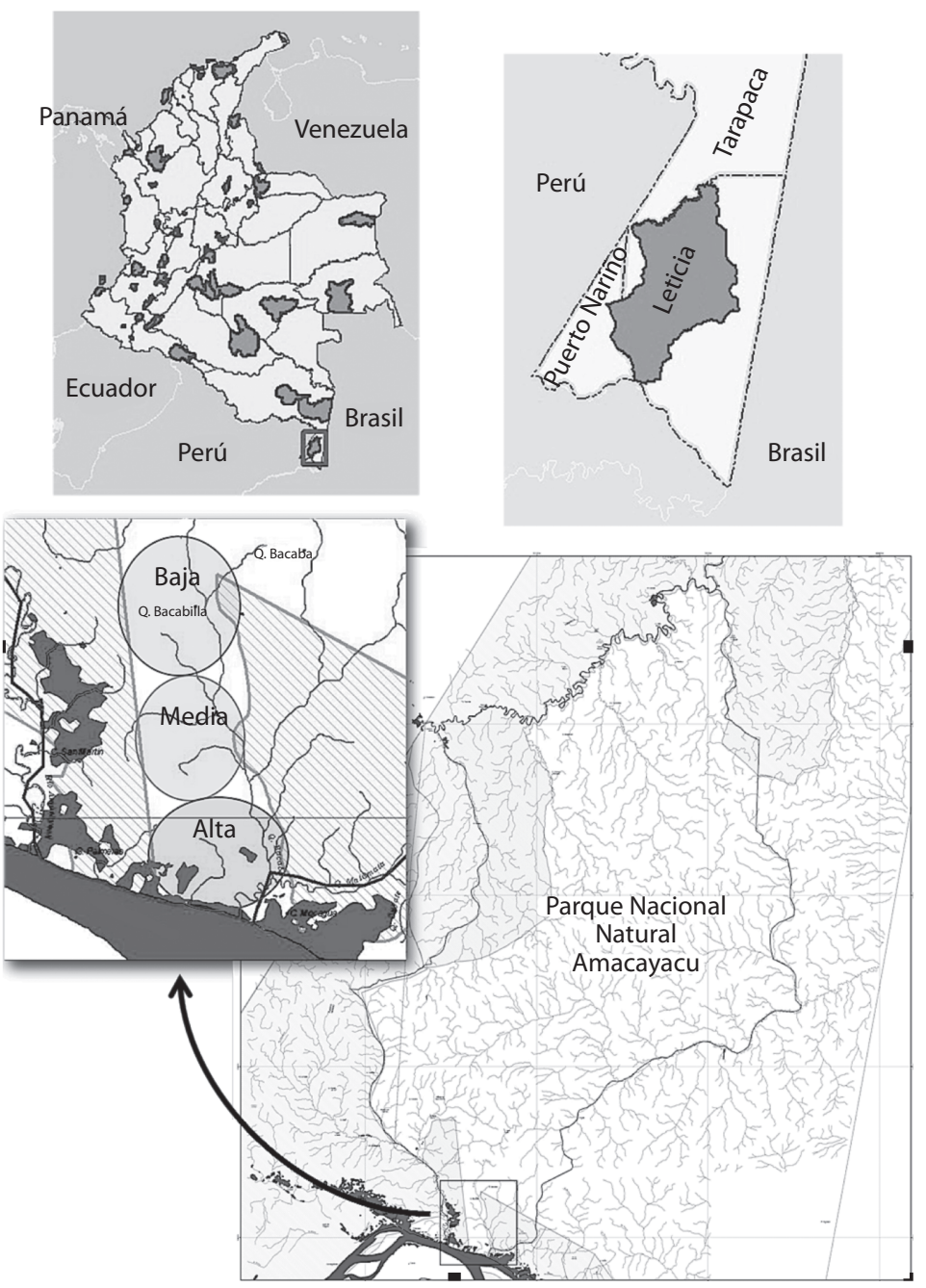

Fig. 1. PNN Amacayacu, Amazonas, Colombia.

Fig. 1. NP Amacayacu, Colombian Amazon.

La zona de baja perturbación (BB) está ubicada a una distancia mayor de $15 \mathrm{~km}$ de las comunidades indígenas $\left(3^{\circ} 40^{\prime} 30^{\prime \prime} \mathrm{S}\right.$ $70^{\circ} 16^{\prime} 32^{\prime}$ W). El único sendero es un camino que comunica con la comunidad de San Martín de Amacayacu, cuya frecuencia de uso es muy baja. La presencia de animales es más evidente debido a la existencia de salados (áreas arenosas donde los vertebrados van a tomar sales) donde se encuentran con cierta frecuencia huellas y excremento de mamíferos. Allí la cacería es muy ocasional con faenas planeadas.
La extracción de madera es nula y la recolección de productos vegetales no maderables es eventual, la cual sucede durante las faenas de cacería (Otavo 2010). Según Gruezmacher \& Duivenvoorden (2008) la cobertura del dosel de BM y BB es similar.

Muestreo de los escarabajos: El muestreo se realizó entre febrero y julio del 2009. Se establecieron cuatro transectos por nivel de perturbación, cada uno de aproximadamente $1600 \mathrm{~m}$ y distanciados uno del otro por $200 \mathrm{~m}$. 
En los tres primeros transectos se instalaron 12 trampas separadas a una distancia de $100 \mathrm{~m}$ entre ellas (adaptado de Morón 1997, Reyes \& Morón 2005, Villarreal et al. 2006, Noriega \& Fagua 2009). El cuarto transecto se utilizó exclusivamente para captura manual, la cual se hizo a través de recorridos ad libitum, durante los cuales se recolectaron los adultos en flores, follaje, hongos, troncos en descomposición y en nidos de insectos sociales (Morón 1997, Carrillo-Ruiz \& Morón 2003, Neita et al. 2006).

Las 12 trampas de cada transecto estaban organizadas de la siguiente forma: a) tres trampas de interceptación al vuelo, cebadas con fruta en descomposición entre los $0-2 \mathrm{~m}$ de altura, en corredores de vuelo para optimizar la efectividad de la captura (Villarreal et al. 2006, Solís 2007); b) Una trampa de luz blanca y otra de luz negra instaladas entre los 10 y $15 \mathrm{~m}$, las cuales funcionaban entre las 16:00 y las 8:00h (Morón 1997, Reyes \& Morón 2005, Neita et al. 2006); c) Cuatro trampas de botella $(1000 \mathrm{~mL})$ cebadas con fruta mezclada en descomposición (plátano, piña y copoazú [Theobroma grandiflorum]), instaladas a 2, 4, 6 y $8 \mathrm{~m}$ de altura (Morón 1997, Reyes \& Morón 2005, Neita et al. 2006, Solís 2007) y d) tres trampas de caída sin cebo (Noriega \& Fagua 2009). El esfuerzo por cada una de las técnicas de captura fue de $48 \mathrm{~h}$ por transecto, excepto para las trampas de luz (16h) y la captura manual (24h). Durante todo el periodo de estudio el esfuerzo de muestreo fue de $536 \mathrm{~h}$ por transecto y $1560 \mathrm{~h}$ por bosque para un total de $4680 \mathrm{~h}$.

Los individuos colectados se montaron, etiquetaron y procesaron en el Laboratorio de Zoología y Ecología Acuática de la Universidad de Los Andes (LAZOEA) y se depositaron en las colecciones de la Universidad Distrital Francisco José de Caldas (UDFJC) y el Museo de Historia Natural de la Universidad de Los Andes (E-ANDES). Estos se identificaron a nivel de especie utilizando claves taxonómicas específicas para cada grupo (Schuster 1993, Morón et al. 1997, Medina \& Lopera 2000, Arnett et al. 2002, Ratcliffe 2003, Edmonds \& Zídek 2004, Reyes \& Morón 2005, Schuster
\& Cano 2005, Triplehorn \& Johnson 2005, Neita et al. 2006, Ocampo 2006, Amat-García \& Reyes-Castillo 2007, González et al. 2009, Camero 2010, Edmonds \& Zídek 2010) y la ayuda de especialistas en cada grupo. La clasificación supragenérica de los grupos taxonómicos se realizó con base en Balthasar (1963).

Para establecer la representatividad del muestreo se utilizaron curvas de acumulación de especies con el software EstimateS 8.2 (Colwell 2000). Por medio del índice de Margalef se analizó la riqueza específica por zona (Magurran 1988, Ramírez 1999, Halffter et al. 2001, Villareal et al. 2006). El índice de Shannon-Wiener $\left(H^{\prime}\right)$ se utilizó para calcular la equidad en la abundancia proporcional (Magurran 1988, Halffter et al. 2001) y el índice de Simpson $(D)$ para calcular el grado de dominancia en la abundancia relativa de las especies (Magurran 1988, Ramírez 1999, Halffter et al. 2001, Villareal et al. 2006).

Para cuantificar la similitud cualitativa entre las diferentes zonas se emplearon los coeficientes de Jaccard $\left(I_{j}\right)$ y Sorensen $\left(I_{s}\right)$ (Krebs 1988). Para el recambio de especies se utilizó el índice de Whittaker (Halffter et al. 2001, Villarreal et al. 2006). La relación entre el grado de perturbación, la abundancia y el número de especies por familia se obtuvo a través del coeficiente de correlación por rangos de Spearman.

Para seleccionar cuáles de los taxones podrían ser considerados como elementos indicadores, se utilizaron dos criterios: que presentaran un patrón a lo largo del gradiente y para el caso de especies, que presentaran un número mayor o igual a 10 individuos por zona. Los análisis de diversidad se realizaron con el programa EstimateS (Colwell 2000) y el programa PAST (Hammer et al. 2001), mientras que los análisis estadísticos se realizaron utilizando el programa SPSS v. 17.0 para Windows.

\section{RESULTADOS}

Composición, riqueza, abundancia y diversidad de la comunidad: Se capturaron 593 individuos agrupados en 92 especies, 44 
géneros y 7 familias pertenecientes a la superfamilia Scarabaeoidea (Cuadro 1). A pesar de la variedad de métodos de captura y el alto esfuerzo de muestreo, las curvas de acumulación para la zona indican un valor medio de eficiencia del muestreo el cual oscila entre el $58 \%$ y el $77 \%$ dependiendo del nivel de intervención (BB: 66-76\%, BM: 58-65\%, BA: 66-77\%; Fig. 2).

Las familias con el mayor número de individuos y especies fueron Scarabaeidae $(n=232$,

\section{CUADRO 1}

Abundancia de las especies de Scarabaeoidea recolectadas en tres niveles de perturbación antropogénica en el PNN Amacayacu, Amazonas, Colombia

TABLE 1

Abundance of Scarabaeoidea species collected in three levels of anthropogenic disturbance in the NP Amacayacu, Colombian Amazon

\begin{tabular}{|c|c|c|c|c|c|c|}
\hline & & Tóxonec & & osque & & \\
\hline & & 1axones & (grado & inter & nción) & Total \\
\hline Subfamilia & Género & Especie & BB & $\mathrm{BM}$ & BA & \\
\hline Cetoniidae & Golinca & G. bifrons Oliver, 1758 & - & - & 1 & 1 \\
\hline & Haplopyga & H. ocellata Gory \& Percheron, 1833 & - & 1 & - & 1 \\
\hline & & Subtotal & - & 1 & 1 & 2 \\
\hline Dynastidae & Aspidolea & A. lindae Ratcliffe, 1978 & - & 1 & 3 & 4 \\
\hline & & A. singularis Bates, 1888 & 1 & 1 & - & 2 \\
\hline & Coelosis & C. biloba (Linnaeus, 1767) & - & - & 3 & 3 \\
\hline & Cyclocephala & C. brevis Perty, 1830 & 2 & 2 & - & 4 \\
\hline & & C. castanea Oliver, 1789 & 1 & - & 1 & 2 \\
\hline & & C. ovulum Bates, 1888 & - & 3 & 1 & 4 \\
\hline & & C. paraflora Martinez, 1878 & - & 1 & - & 1 \\
\hline & & C. pugnax Arrow, 1914 & - & - & 3 & 3 \\
\hline & & C. rufovaria Arrow, 1911 & - & 1 & - & 1 \\
\hline & & C. santaritae Ratcliffe, 1992 & 1 & - & 1 & 2 \\
\hline & & C. verticalis Burmeister, $1847^{*}$ & 7 & 26 & 57 & 90 \\
\hline & & C. sp. 1 & - & - & 1 & 1 \\
\hline & & C. sp. 2 & - & 1 & - & 1 \\
\hline & & C. sp. 3 & 1 & - & - & 1 \\
\hline & Dyscinetus & D. dubius (Oliver, 1789) & - & 1 & 1 & 2 \\
\hline & Erioscelis & E. sp. & - & - & 1 & 1 \\
\hline & Homophileurus & H. quadrituberculatus Palisot de Beauvois, 1806 & - & - & 1 & 1 \\
\hline & Ligyrus & L. bituberculatus (Beauvois, 1805) & - & - & 2 & 2 \\
\hline & & L. gyas Erichson, 1848 & - & - & 11 & 11 \\
\hline & & L. similis Endrödi, 1968 & - & - & 1 & 1 \\
\hline & Megaceras & M. crassum Prell, 1914 & - & - & 2 & 2 \\
\hline & Stenocrates & S. cf. popei Endrödi, 1971 & - & - & 1 & 1 \\
\hline & & S. minutus Endrödi, 1967 & - & 5 & 2 & 7 \\
\hline & & S. hiekei Endrödi, 1967 & - & 2 & 3 & 5 \\
\hline & & S. sp. & - & - & 8 & 8 \\
\hline & Surutus & S. dityscoides Martínez, 1955 & - & - & 1 & 1 \\
\hline & & Subtotal & 13 & 44 & 104 & 161 \\
\hline Melolonthidae & Astaena & $A$. sp. & 22 & - & - & 22 \\
\hline & Barybas & $B$. sp. & 2 & - & - & 2 \\
\hline & Isonychus & I. sp. 1 & 1 & - & 1 & 2 \\
\hline & & I. sp. 2 & - & 1 & - & 1 \\
\hline
\end{tabular}


CUADRO 1 (Continuación)

Abundancia de las especies de Scarabaeoidea recolectadas en tres niveles de perturbación antropogénica en el PNN Amacayacu, Amazonas, Colombia

TABLE 1 (Continued)

Abundance of Scarabaeoidea species collected in three levels of anthropogenic disturbance in the NP Amacayacu, Colombian Amazon




CUADRO 1 (Continuación)

Abundancia de las especies de Scarabaeoidea recolectadas en tres niveles de perturbación antropogénica en el PNN Amacayacu, Amazonas, Colombia

TABLE 1 (Continued)

Abundance of Scarabaeoidea species collected in three levels of anthropogenic disturbance in the NP Amacayacu, Colombian Amazon

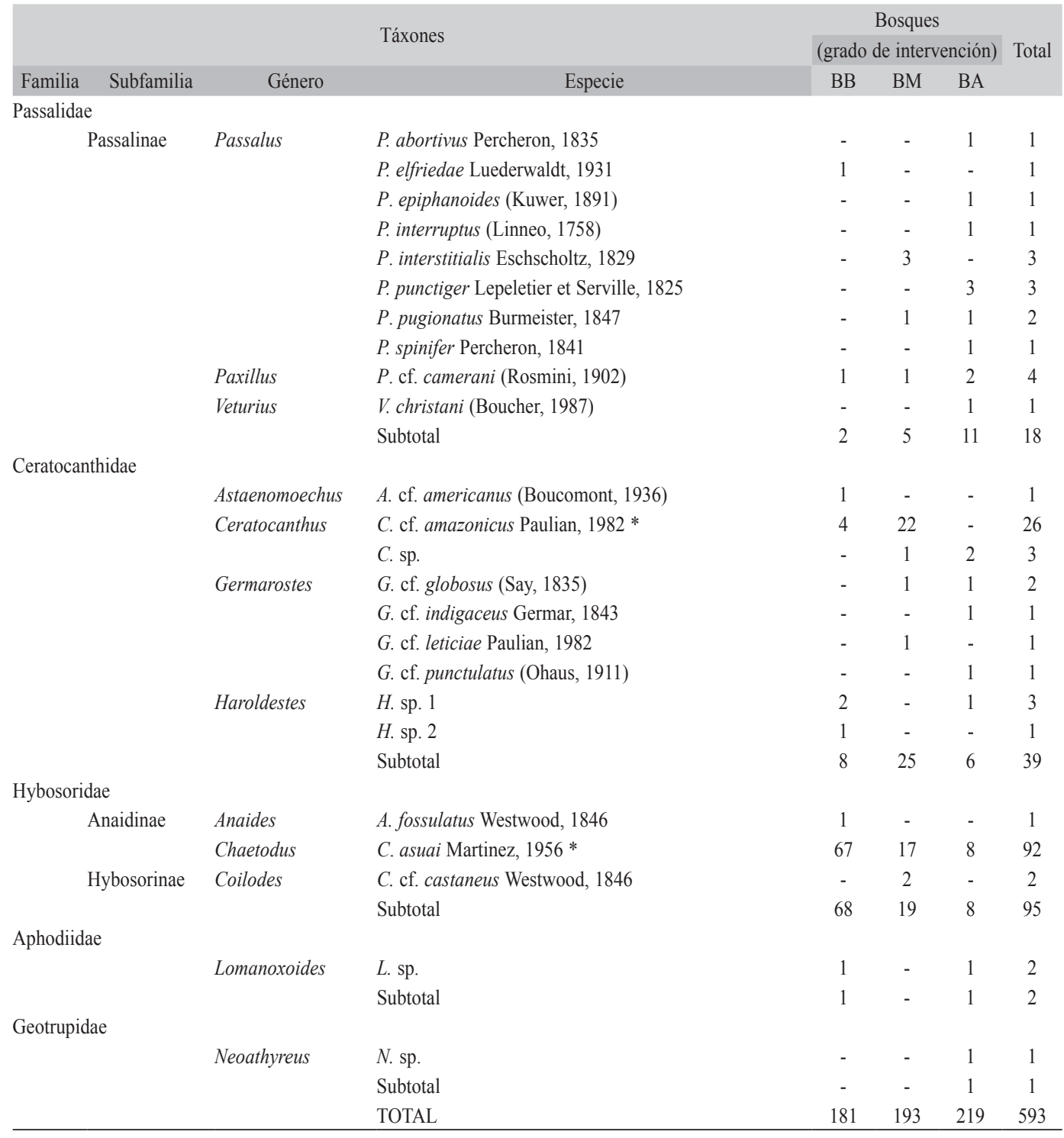

*=especies con más de 10 individuos.

*=species with more than 10 individuals.

BB: Bosque baja perturbación, BM: Bosque media perturbación y BA: Bosque alta perturbación.

BB: Low disturbed forest, BM: Medium disturbed forest, BA: High disturbed forest. 

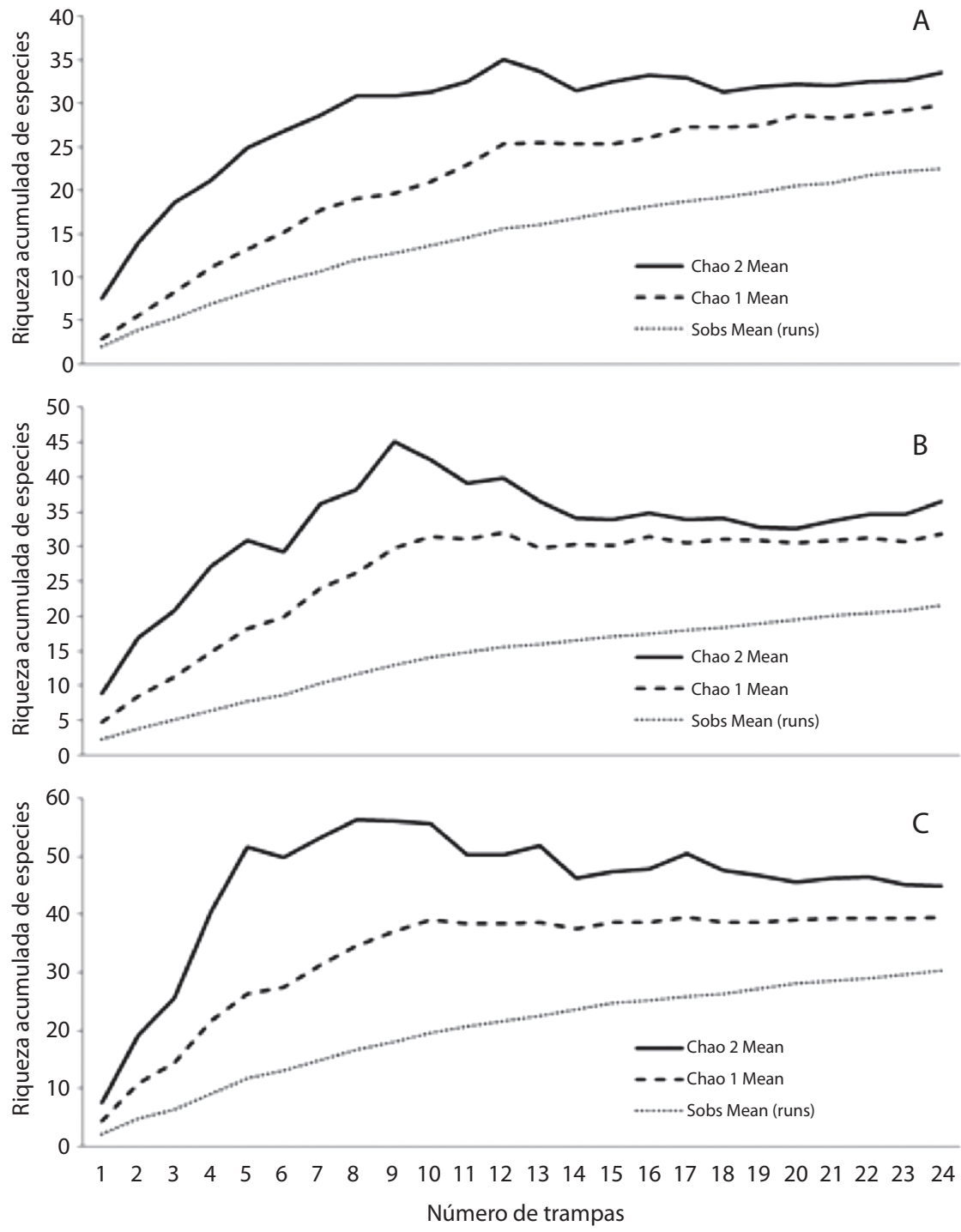

Fig. 2. Curva de acumulación de especies de Scarabaeoidea en tres niveles de perturbación antropogénica en el PNN Amacayacu, Amazonas, Colombia. A) BB: Bosque baja perturbación, B) BM: Bosque media perturbación y C) BA: Bosque alta perturbación.

Fig. 2. Species accumulation curve of Scarabaeoidea collected in three levels of anthropogenic disturbance in the NP Amacayacu, Colombian Amazon. A) BB: Low disturbed forest, B) BM: Medium disturbed forest and C) BA: High disturbed forest.

27 spp.) y Dynastidae (n=161, 25 spp.) (Cuadro 1, Fig. 3). Las familias Aphodiidae $(\mathrm{n}=2,1$ sp.), Cetoniidae ( $\mathrm{n}=2,2$ spp.) y Geotrupidae $(\mathrm{n}=1,1$ sp.) presentaron un valor muy bajo tanto de especies como de individuos, siendo poco representativas en los análisis. Scarabaeidae presentó nueve géneros siendo Ateuchus el más abundante, seguido de Eurysternus y Canthidium los cuales se encuentraron presentes en los tres niveles de intervención (Cuadro 1). En la familia Dynastidae se encontraron 10 géneros siendo Cyclocephala el más abundante y rico en número de especies. En cuanto a las especies más dominantes por familia se 


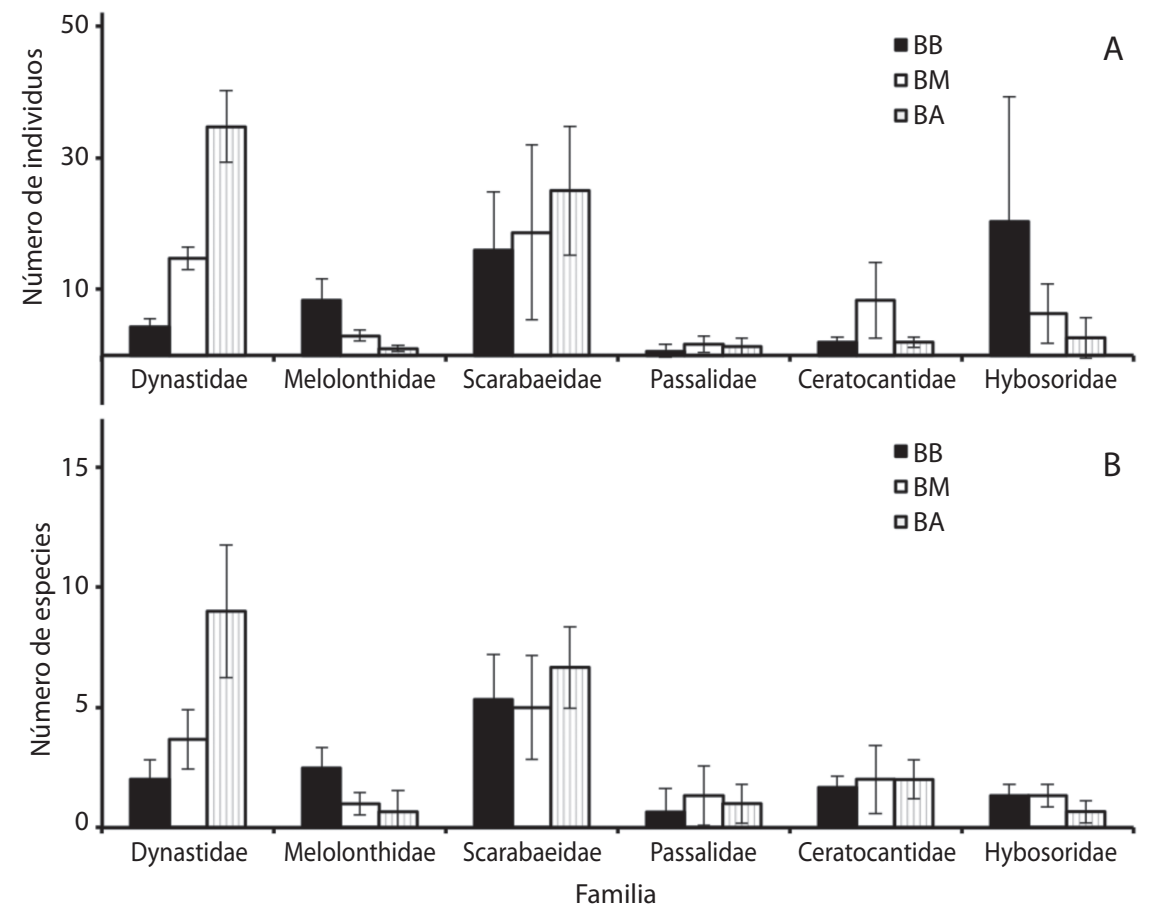

Fig. 3. Número total de individuos (A) y número total de especies (B) por familia de Scarabaeoidea encontrados en cada uno de los niveles de perturbación antropogénica en el PNN Amacayacu, Amazonas, Colombia. BB: Bosque baja perturbación, BM: Bosque media perturbación y BA: Bosque alta perturbación.

Fig. 3. Total number of Scarabaeoidea individuals (A) and species (B) per family recorded in each level of anthropogenic disturbance in the NP Amacayacu, Colombian Amazon. BB: Low disturbed forest, BM: Medium disturbed forest and BA: High disturbed forest.

encuentra Ateuchus sp. 2 (33.2\%) para Scarabaeidae, Cyclocephala verticalis (43.7\%) para Dynastidae, Astaena sp. (75.8\%) para Melolonthidae, Ceratocanthus cf. amazonicus (66.7\%) para Ceratocanthidae y Chaetodus asuai (96.8\%) para Hybosoridae (Cuadro 1).

Gradiente de perturbación: En términos generales el número de especies e individuos fue mayor a medida que el gradiente de perturbación antropogénica aumentó (Cuadro 2). Aunque este patrón no fue estadísticamente significativo (Coeficiente de correlación por Rangos de Spearman, Especies $r_{s}=0.467$, $\mathrm{p} \geq 0.05$; Individuos $r_{s}=0.250, p \geq 0.05$ ). Los índices de Margalef y Shannon señalan que BA presenta la mayor riqueza y equidad, respectivamente (Cuadro 2). El índice de Simpson,

\section{CUADRO 2}

Número de especies (riqueza), individuos (abundancia) e índices de diversidad de Scarabaeoidea en tres niveles de perturbación antropogénica en el PNN Amacayacu, Amazonas, Colombia. BB: Bosque baja perturbación, BM: Bosque media perturbación y BA: Bosque alta perturbación

TABLE 2

Number of species (richness), individuals (abundance) and diversity index of Scarabaeoidea in three levels of anthropogenic disturbance in the NP Amacayacu, Colombian Amazon. BB: Low disturbed forest, BM: Medium disturbed forest, BA: High disturbed forest

\begin{tabular}{lccc} 
& BB & BM & BA \\
No. especies & 36 & 44 & 58 \\
No. individuos & 181 & 193 & 219 \\
Margalef (d) & 6.733 & 8.171 & 10.58 \\
Shannon-Wiener $\left(H^{\prime}\right)$ & 2.507 & 3.015 & 3.042 \\
Simpson (D) & 0.826 & 0.926 & 0.892 \\
\hline
\end{tabular}


CUADRO 3

Índices de similitud de Jaccard $\left(\mathrm{I}_{\mathrm{j}}\right)$ y Sorensen $\left(\mathrm{I}_{\mathrm{s}}\right.$ ) en tres niveles de perturbación antropogénica en el PNN Amacayacu, Amazonas, Colombia. BB: Bosque baja perturbación, BM: Bosque media perturbación y BA: Bosque alta perturbación

TABLE 3

Jaccard $\left(\mathrm{I}_{\mathrm{j}}\right)$ y Sorensen $\left(\mathrm{I}_{\mathrm{s}}\right)$ similarity indexes in three levels of anthropogenic disturbance in the NP Amacayacu, Colombian Amazon. BB: Low disturbed forest, BM: Medium disturbed forest, BA: High disturbed forest

\begin{tabular}{lccccccccc}
\hline \multicolumn{3}{r}{ Jaccard } & \multicolumn{3}{c}{ General } & \multicolumn{3}{c}{ Dynastidae } & \multicolumn{3}{c}{ Scarabaeidae } \\
Sorensen & BB & BM & BA & BB & BM & BA & BB & BM & BA \\
BB & & 0.290 & 0.205 & & 0.160 & 0.118 & & 0.500 & 0.348 \\
BM & 0.450 & & 0.275 & 0.276 & & 0.216 & 0.667 & & 0.391 \\
BA & 0.340 & 0.431 & & 0.211 & 0.356 & & 0.516 & 0.563 & \\
\hline
\end{tabular}

por el contrario, muestra que la dominancia fue más alta en BM (1- $\lambda=0.926$; Cuadro 2).

Según los índices de similitud de Jaccard y Sorensen, BB y BA son disímiles tanto para toda la superfamilia como a nivel de las familias más representativas (Dynastidae y Scarabaeidae; Cuadro 3). Así, la familia Dynastidae contiene el mayor número de especies exclusivas por gradiente mientras que la familia Scarabaeidae es la que comparte más especies (Cuadro 3). Estas diferencias también se reflejan al evaluar la tasa de recambio de especies, siendo la mayor entre BA y BB (Whittaker=0.66), mientras que la tasa de recambio entre $\mathrm{BM}$ y $\mathrm{BA}$ (Whittaker $=0.57$ ) y $\mathrm{BB}$ y $\mathrm{BM}$ es menor (Whittaker=0.55).

Al analizar el número de especies y de individuos por familia a lo largo del gradiente, se encuentra que para Dynastidae y Scarabaeidae estos valores aumentan a medida que la perturbación es mayor (Fig. 3). No obstante, el patrón no es significativo, pues las diferencias entre BB y BM son mínimas (Dynastidae especies, $r_{s}=0.346, p \geq 0.05$; individuos, $r_{s}=0.350$, $\mathrm{p} \geq 0.05 ; \quad$ Scarabaeidae especies, $\mathrm{r}_{\mathrm{s}}=0.333$ $\mathrm{p} \geq 0.05$; individuos, $\mathrm{r}_{\mathrm{s}}=0.363, \mathrm{p} \geq 0.05$ ). En contraste, la familia Hybosoridae y Melolonthidae presentan una disminución del número de individuos a medida que el grado de perturbación aumenta, siendo significativa para Hybosoridae $\left(r_{s}=0,692, p \leq 0.05\right)$, pero no para el número de especies $\left(r_{\mathrm{s}}=0.175, \mathrm{p}>0.05\right.$; Fig. 3$)$.

Potenciales elementos indicadores: Diez especies se registraron en los tres niveles de perturbación, 26 en dos y 56 fueron exclusivas para un sólo nivel de perturbación. BA tiene un $55.4 \%$ de especies exclusivas mientras que BM presenta un $23.2 \%$ y BB un $21.4 \%$. Por otro lado, de las 11 especies que presentaron el mayor número de individuos ( $\mathrm{n}>10$; Cuadro 1) se encuentra que Chaetodus asuai (Hybosoridae) fue la más importante, seguida de Cyclocephala verticalis (Dynastidae), ambas presentes en todos los niveles de perturbación. Las especies Astaena sp. (Melolonthidae) y $L$. gyas (Dynastidae) fueron exclusivas a un sólo bosque (BB y BA, respectivamente).

De estas especies, $C$. verticalis muestra que a medida que aumenta la perturbación antropogénica, el número de individuos se incrementa significativamente $\left(r_{s}=0,683\right.$, $\mathrm{p}<0.005)$. En contraste, para C. asuai el número de individuos disminuye de forma significativa a medida que aumenta la perturbación $\left(\mathrm{r}_{\mathrm{s}}=0.642, \mathrm{p}<0.05\right)$. Con excepción de L. gyas y Astaena sp. que sólo se registraron en un nivel de perturbación, las siete especies restantes no presentaron patrones evidentes.

\section{DISCUSIÓN}

Riqueza y diversidad de la comunidad: La riqueza de especies aumentó a medida que la perturbación fue mayor, lo cual contrasta con una gran cantidad de estudios en los que una mayor riqueza y/o diversidad se asocia a una mayor complejidad ecosistémica correspondiente a menores niveles de perturbación 
(Klein 1989, Escobar \& Chacón 2000, Celi \& Dávalos 2001, Davis et al. 2001, Halffter \& Arellano 2002, Arellano et al. 2004, Escobar 2004, García \& Pardo 2004, Scheffler 2005, Vidaurre et al. 2008). Posiblemente, los mayores valores de diversidad en el bosque de alta perturbación encontrados en el presente estudio, pueden ser consecuencia del arribo de especies generalistas o especies de zonas abiertas que tienen la capacidad de reemplazar y desplazar especies asociadas a las zonas más conservadas del bosque (Yahner 1988, Davis \& Sutton 1998, Halffter \& Arellano 2002, Herrera-Montes et al. 2004, Pineda et al. 2005, Noriega et al. 2007a).

Igualmente, las diferencias mencionadas podrían atribuirse a que la mayoría de los trabajos realizados en coleópteros como indicadores se han enfocado principalmente en escarabajos coprófagos (Scarabaeidae), mientras que al incluir a toda la superfamilia, se incluyen táxones con diferentes niveles de sensibilidad ante las perturbaciones, que al parecer no presentan el mismo patrón de sensibilidad. En el caso particular de la familia Scarabaeidae, esta fue la más abundante y también presentó una mayor riqueza (aunque no significativa) en el bosque de mayor perturbación. Sin embargo, es importante aclarar que las trampas de caída utilizadas no fueron cebadas con excremento humano (como normalmente se hace), lo cual estaría influyendo en las abundancias de las especies de escarabajos coprófagos al ser menos atractivas. Por lo tanto, teniendo en cuenta que este grupo ha sido sugerido como indicador de diferencias estructurales entre ecosistemas, sería importante incluir esta metodología específica de muestreo en futuros estudios, así como profundizar en el estudio del BM que presentó una mayor abundancia y diversidad y podría estar funcionando como un corredor biológico entre BA y BB.

Con base en lo anterior, la ausencia de una relación directa entre la diversidad y la riqueza con el nivel de perturbación, corrobora que estas variables no son apropiadas como indicadoras de integridad ecológica de un sitio, tal y como lo sugiere Noss (1990). Esto también soporta el trabajo realizado con anterioridad por el Sistema de Parques Nacional Naturales de Colombia, sobre la obtención de una metodología para el análisis de la integridad ecológica en sus áreas, pues se descartó la diversidad como indicador, por los posibles ruidos que pueden generar especies invasoras y aquellas típicas de zonas perturbadas (Zambrano et al. 2007). En contraste, la abundancia de taxones particulares si resultó efectiva para medir la respuesta de los bosques ante las perturbaciones (Barlow et al. 2007, Nichols et al. 2007, Basset et al. 2008).

Potenciales elementos indicadores: Los datos encontrados en este estudio ayudan a soportar la evidencia existente que señala a los coleópteros como importantes elementos indicadores del estado de conservación de un hábitat (Morón 1997, Anderson \& Ashe 2000, Schuster et al. 2000, Basset et al. 2004, UeharaPrado et al. 2009, Schulze et al. 2004, Barlow et al. 2007, Basset et al. 2008). En este caso en particular, algunos taxa respondieron de forma diferencial a la perturbación antropogénica en los bosques amazónicos de tierra firme del PNN Amacayacu. Las especies $C$. verticalis, $C$. asuai, L. gyas y Astaena sp. mostraron un claro patrón a lo largo del gradiente de perturbación, lo cual puede ser bastante prometedor en la búsqueda de posibles elementos indicadores de los bosques amazónicos de tierra firme. Sin embargo, es importante señalar que la totalidad de la superfamilia no puede ser utilizada como un único elemento bioindicador debido a las grandes diferencias que existen en sus componentes (familias, subfamilias, géneros y especies) y a la respuesta diferencial que tienen los distintos componentes (e.j. Dynastidae vs. Hybosoridae) a los eventos de perturbación.

La menor abundancia de $C$. asuai y la ausencia de Astaena sp. en BA, podrían sugerir una simplificación de la complejidad estructural y el deterioro de la integridad de los bosques de tierra firme. En contraste, el aumento de $C$. verticalis estaría asociado a zonas más abiertas y con menos competidores. Adicionalmente, el gran número de especies únicas de la familia 
Dynastidae asociadas a un nivel particular de perturbación, puede ser una señal de una alta especificidad de hábitat (Morón 2009).

Por otro lado, aunque no significativo, el número de individuos y especies de las familias Dynastidae, Melolonthidae, Scarabaeidae e Hybosoridae mostró una tendencia a lo largo del patrón. Para la familia Hybosoridae y Melolonthidae el número de individuos disminuyó a medida que la perturbación aumentó (principalmente por la presencia de C. asuai y Astaena sp.). Para Dynastidae, Passalidae y Scarabaeidae el número de especies y de individuos aumentó a medida que aumentó la perturbación, especialmente para C. verticalis. Dynastidae podría ser un potencial elemento indicador. Por lo tanto, futuros estudios podrían enfocarse exclusivamente en esta familia, con trampas más específicas para este grupo, con el fin de corroborar esta tendencia.

Implicaciones para la medición de la integridad ecológica: La selección de táxones como elementos indicadores de integridad ecológica debe tener criterios de soporte, tanto biológicos como logísticos, pues debe generar la mejor resolución posible en un corto tiempo de muestreo, permitiendo el poder tomar decisiones efectivas y precisas (Fernández 2003). En este contexto, los táxones identificados cumplen con las condiciones necesarias que debe presentar un grupo bioindicador según Pearson (1994). Sin embargo, aún se desconoce gran parte de sus historias de vida, por lo que futuros estudios deben profundizar en su biología y ecología, con el fin de avalar su papel como elementos indicadores de integridad ecológica de los bosques amazónicos y en especial en el caso particular del PNN Amacayacu. Adicionalmente, teniendo en cuenta la sensibilidad de las especies, la intensidad del muestreo, la escala espacial y temporal del estudio y especialmente la variación estacional en la oferta de recursos alimenticios que puede condicionar la riqueza y abundancia de las especies, es fundamental realizar réplicas en otras épocas del año y en otros bosques de tierra firme con el fin de validar estos resultados.
Uno de los aspectos que mayores complicaciones presentó a la hora de identificar elementos indicadores fue la determinación taxonómica a nivel de especie, dada la alta diversidad y el nivel de desconocimiento de la superfamilia para esta región de Colombia en comparación con otras zonas del país (García \& Pardo 2004, Neita et al. 2006, CarvajalCogollo \& Urbina-Cardona 2008, Noriega et al. 2007b, 2008). Aunque se contó con la colaboración de expertos en cada uno de los grupos, no siempre fue fácil contar con estas facilidades. Por lo tanto, si se desean incluir los grupos sugeridos en las estrategias de monitoreo de los parques amazónicos habría que evaluar el nivel de identificación taxonómica al que se puede llegar por parte de funcionarios y técnicos de la región, y/o en caso dado, hacer alianzas con instituciones de investigación que faciliten las labores de identificación de las especies, pues aunque es cuestión de debate, se ha sugerido que a mayor especificidad la resolución es mayor (Lenat \& Resh 2001).

Finalmente, aunque el PNN Amacayacu fue creado y delimitado en 1975, este trabajo muestra que hoy en día aun existen diferencias notables en la composición y estructura del bosque que no se han logrado subsanar a pesar de las medidas de conservación y protección del sitio. Esto hace pensar que los bosques amazónicos son muy sensibles a las perturbaciones y su recuperación es extremadamente lenta. El diseño de futuros planes de monitoreo dirigido a los grupos y las especies identificadas en el presente estudio, como posibles indicadores serviría en el análisis de integridad ecológica del PNN Amacayacu, ya que cambios en su composición, abundancia y riqueza se podrían traducir en el cumplimiento o no de los objetivos de conservación y restauración establecidos.

\section{AGRADECIMIENTOS}

Agradecemos a la Fundación Alejandro Ángel Escobar y a su fondo Colombia Biodiversa, al Centro de Investigaciones y Desarrollo Científico de la Universidad Distrital 
Francisco José de Caldas, así como a las Fundaciones Idea Wild y Tropenbos International Colombia por el apoyo económico y de equipos. Al Laboratorio de Zoología y Ecología Acuática-LAZOEA de la Universidad de Los Andes por el préstamo de las instalaciones. A la Unidad Administrativa Especial del Sistema de Parques Nacionales Naturales de Colombia, por el apoyo logístico en campo, en especial a su director Alexander Alonso. A las comunidades Indígenas por su apoyo en campo principalmente a Bartolomé Morán. A Miguel Ángel Morón, Jhon Cesar Neita, Fernando Vazde-Mello, Jason Mate, Carlos Aguilar, Alberto Ballerio, Federico Ocampo, Paul Scholmeesters, Jean Bernard Huchet, Marco Dellacasa, Zdzislawa Teresa Stebnicka, Pedro Reyes y Jack Schuster por su ayuda en la identificación taxonómica. A ScarabNet por su valiosa contribución en mejorar y establecer eficientes mecanismos de comunicación entre investigadores. A Patricia Pinzón, Max Triana, Daniel Monroy, Julián Monge Nájera y a dos evaluadores anónimos por sus comentarios y críticas a diferentes versiones de este documento.

\section{RESUMEN}

Diferentes grupos de insectos se consideran importantes elementos bioindicadores de la calidad de los ecosistemas, los coleópteros son hoy en día uno de los grupos más reconocidos y utilizados para el monitoreo y establecimiento de áreas protegidas. Se evaluó el uso de la superfamilia Scarabaeoidea como bioindicador del grado de perturbación antropogénica de los bosques húmedos amazónicos. En tres niveles de perturbación (alta, media y baja) se establecieron 12 transectos, utilizando tres trampas de interceptación, dos trampas de luz, tres trampas de caída, cuatro trampas de botella y captura manual. Se capturaron 593 individuos, 92 especies, 44 géneros y 7 familias. Las familias con el mayor número de individuos y especies fueron Scarabaeidae ( $\mathrm{n}=232,27 \mathrm{spp}$.) y Dynastidae (n=161, 26 spp.). Las especies más abundantes fueron: Ateuchus sp., Cyclocephala verticalis, Ceratocanthus amazonicus y Chaetodus asuai. El número de especies e individuos fue mayor a medida que el gradiente de perturbación aumentó. Dynastidae presenta el mayor número de especies exclusivas mientras Scarabaeidae comparte la mayoría. Diez especies fueron registradas en los tres bosques, 26 en dos y 56 en solo uno. De las especies más abundantes $C$. asuai muestra una reducción significativa del número de individuos con el aumento de la perturbación, mientras se observa una relación inversa para $C$. verticalis. Se discute la utilización de la riqueza y la diversidad como indicadores de perturbación, mientras se ratifica la abundancia de ciertos táxones como una mejor variable a medir.

Palabras clave: Amazonía, Dynastidae, escarabajos, Hybosoridae, indicadores, integridad ecológica, Scarabaeidae.

\section{REFERENCIAS}

Amat, G.G., A.T. Lopera \& S. Amézquita. 1997. Patrones de distribución de escarabajos coprófagos en relicto del bosque alto andino Cordillera Oriental de Colombia. Caldasia 19: 191-204.

Amat, G.G., E. Blanco \& P. Reyes-Castillo. 2004. Lista de especies de los escarabajos pasálidos (Coleoptera:Passalidae) de Colombia. Biota Col. 5: 173-182.

Amat-García, G. \& P. Reyes-Castillo. 2007. Los Passalidae (Coleoptera: Scarabaeoidea) del departamento del Amazonas, Colombia. Caldasia 29: 329-354.

Amézquita, M.S., A. Forsyth, A. Lopera \& A. Camacho. 1999. Comparación de la composición y riqueza de especies de escarabajos coprófagos (Coleoptera: Scarabaeidae) en remanentes de bosque de la Orinoquía Colombiana. Acta Zool. Mex. 76: 113-126.

Anderson, R.S. \& J.S. Ashe. 2000. Leaf litter inhabiting beetles as surrogates for establishing priorities for conservation of selected tropical montane cloud forests in Honduras, Central America (Coleoptera Staphylinidae, Curculionidae). Biodiversity Conserv. 9: 617-653.

Arnett, R.H., M.C. Thomas, P.E. Skelley \& J.H. Frank. 2002. American Beetles. CRC, Boca Raton, Florida, EE.UU.

Arellano, L., M.E. Favila \& C. Huerta. 2004. Diversity of dung and carrion beetles in a disturbed Mexican tropical montane cloud forest and on shade coffee plantations. Biodiversity Conserv. 14: 601-615.

Balthasar, V. 1963. Monographie der Scarabaeidae und Aphodiidae der Paläarktischen und Orientalischen Region (Coleoptera: Lamellicornia). Band 1-3. Tschechoslowak Akademie der Wissenschaften, Praha.

Barlow, J., T.A. Gardner, I.S. Araujo, T.C. Avila-Pires, A.B. Bonaldo, J.E. Costa, M.C. Esposito, L.V. Ferreira, J. Hawes, M.M. Hernandez, M.S. Hoogmoed, R.N. Leite, N.F. Lo-Man-Hung, J.R. Malcolm, M.B. Martins, L.A.M. Mestre, R. Miranda-Santos, A.L. Nunes-Gutjahr, W.L. Overal, L. Parry, S.L. Peters, M.A. Ribeiro-Junior, M.N.F. da Silva, C.S. Motta \& C.A. Peres. 2007. Quantifying the biodiversity value of tropical primary, secondary, and plantation forests. Proc Natl Acad Sci USA 104: 18555-18560. 
Basset, Y, J. Mavoungou, J.B. Mikissa, O. Missa, S.E. Miller, R.L. Kitching \& A. Alonso. 2004. Discriminatory power of different arthropod data sets for the biological monitoring of anthropogenic disturbance in tropical forests. Biodiversity Conserv. 13: 709-732.

Basset, Y., O. Missa, A. Alonso, S.E. Miller, G. Curletti, M. De Meyer, C. Eardley, O.T. Lewis, N.W. Mansell, V. Novotny \& T. Wagner. 2008. Choice of metrics for studying arthropod responses to habitat disturbance: one example from Gabon. Insect Conserv. Diversity 1: 55-66.

Camero, E. 2010. Los escarabajos del género Eurysternus Dalman, 1824 (Coleoptera: Scarabaeidae) de Colombia. Boletín S.E.A. 46: 147-179.

Campos, D. \& F. Fernández. 2002. El Proyecto "Diversidad de Insectos de Colombia". C. Costa, S.A. Vanin, J.M. Lobo \& A. Melic. 2002. Proyecto de Red Iberoamericana de Biogeografía y Entomología Sistemática PrIBES. 2002. Monografías Tercer Milenio, Zaragoza, España. 2: 297-300.

Carignan, V. \& M.A. Villard. 2002. Selecting indicator species to monitor ecological integrity: a review. Environ. Monitor. Assess. 78: 45-61.

Caro, T., J. Eadie \& A. Sih. 2005. Use of substitute species in conservation biology. Conserv. Biol. 19: 1821-1826.

Carrillo-Ruiz, H. \& M.A. Morón. 2003. Fauna de Coleoptera Scarabaeoidea de Cuetzalan del Progreso, Puebla, México. Acta Zool. Mex. 88: 87-121.

Carvajal-Cogollo, J.E. \& J.N. Urbina-Cardona. 2008. Patrones de Diversidad y Composición de Reptiles en Fragmentos de Bosque Seco Tropical en Córdoba, Colombia. Trop. Conserv. Sci. 1: 397-416.

Celi, J. \& A. Dávalos. 2001. Manual de Monitoreo. Los escarabajos peloteros como indicadores de la calidad ambiental. EcoCiencia, Quito, Ecuador.

Concha, C., M. Gallego \& L.C. Pardo-Locarno. 2010. Fragmentación de ecosistemas montanos e impactos estructurales y poblacionales sobre la comunidad de escarabajos coprófagos (Coleoptera: Scarabaeidae) en el alto Río Cauca, Popayán, Colombia. Bol. Cien. Mus. Hist. Nat. 14: 43-55.

Colwell, R.K. 2000. Estimates: Statistical estimation of species richness and shared species from samples. Storrs, Connecticut, EEUU:

Dale, V.H. \& S.C. Beyeler. 2001. Challenges in the development and use of ecological indicators. Ecol. Indicators 1: 3-10.

Davis, A.J. \& S.L. Sutton. 1998. The effects of rainforest canopy loss on arboreal dung beetles in Borneo: implications for the measurement of biodiversity in derived tropical ecosystems. Diversity and Distributions 4: 167-173.
Davis, A.J., J.D. Holloway, H. Huijbregts, J. Krikken, A.H. Kirk-Springgs \& S.L. Sutton. 2001. Dung beetles as indicators of change in the forests of northern Borneo. J. Appl. Ecol. 38: 593-616.

Edmonds, W.D. \& J. Zídek. 2004. Revision of the Neotropical dung beetle genus Oxysternon (Scarabaeidae: Scarabaeinae: Phanaeini). Folia Heyrovskyana 11: $1-58$.

Edmonds, W.D. \& J. Zídek. 2010. A taxonomic review of the neotropical genus Coprophanaeus Olsoufieff, 1924 (Coleoptera: Scarabaeidae, Scarabaeinae). Insecta Mundi. 0129: 1-111.

Escobar, F. 1997. Estudio de la comunidad de coleópteros coprófagos (Scarabaeidae) en un remanente de bosque seco al norte del Tolima, Colombia. Caldasia 19: 419-430.

Escobar, F. 2000. Diversidad de coleópteros coprófagos (Scarabaeidae: Scarabaeinae) en un mosaico de hábitats en la Reserva Natural Nukak, Guaviare, Colombia. Acta Zool. Mex. 79: 103-121.

Escobar, F. \& P. Chacon. 2000. Distribución espacial y temporal en un gradiente de sucesión de la fauna de coleópteros coprófagos (Scarabaeinae, Aphodiinae) en un bosque tropical montano, Nariño-Colombia. Rev. Biol. Trop. 48: 961-975.

Escobar, F. 2004. Diversity and composition of dung beetle (Scarabaeinae) assemblages in a heterogeneous Andean landscape. Trop. Zool. 17: 123-136.

Fernández, F. 2003. Introducción a las Hormigas de la región Neotropical. Instituto de Investigación de Recursos Biológicos Alexander Von Humboldt, Bogotá. Colombia.

García, J.C. \& L. Pardo. 2004. Escarabajos Scarabaeinae saprófagos (Coleóptera: Scarabaeidae) en un Bosque muy Húmedo Premontano de los Andes Occidentales Colombianos. Ecol. Aplicada 3: 59-63.

Gardner, T.A., J. Barlow, I.S. Araujo, T.C. Ávila-Pires, A.B. Bonaldo, J.E. Costa, M.C. Esposito, L.V. Ferreira, J. Hawes, M.I.M. Hernandez, M.S. Hoogmoed, R.N. Leite, Lo-N.F. Man-Hung, J.R. Malcolm, M.B. Martins, L.A.M. Mestre, R. Miranda-Santos, W.L. Overal, L. Parry, S.L. Peters, M.A. Ribeiro-Junior, M.N.F. da Silva, C.S. Motta \& C.A. Peres. 2008. The cost-effectiveness of biodiversity surveys in tropical forests. Ecol. Lett. 11: 139-150.

Gruezmacher, M. \& J.F. Duivenvoorden. 2008. Growth of transplanted timber species seedlings in the south of the Colombian Amazon: a preliminary study. Col. Forestal. 11: 35-43.

González, F.A., F. Molano \& C.A. Medina. 2009. Los subgéneros Calhyboma, Hybomidium y Telhyboma (Coleoptera: Scarabaeidae: Scarabaeinae: Deltochilum) en Colombia. Rev. Col. Entomol. 35: 253-274. 
Halffter, G. \& M.E. Favila. 1993. The Scarabaeidae (Insecta: Coleoptera) an animal group for analysing, inventoryng and monitoring biodiversity in tropical rainforest and modified landscapes. Biol. Int. 27: $1-21$.

Halffter, G., C.E. Moreno \& E.O. Pineda. 2001. Manual para evaluación de la biodiversidad en Reservas de la Biosfera. Manuales y Tesis SEA, vol. 2. Zaragoza, España.

Halffter, G. \& L. Arellano. 2002. Response of dung beetle diversity to human-induced changes in a tropical landscape. Biotropica 34: 144-154.

Hammer, O., D.A.T. Harperand \& P.D. Ryan. 2001. PAST: Paleontological Statistics Software Package for Education and Data Analysis. Paleontología Electrónica 4: 9.

Herrera-Montes, A., L.A. Olaya-M \& F. Castro-H. 2004. Incidencia de la perturbación antrópica en la diversidad, la riqueza y la distribución de Eleutherodactylus (Anura: Leptodactylidae) en un bosque nublado del suroccidente colombiano. Caldasia 26: 265-274.

Hodkinson, I.D. \& J.K. Jackson. 2005. Terrestrial and aquatic invertebrates as bioindicators for environmental monitoring, with particular reference to mountain ecosystems. Enviro. Management. 35: 649-666.

Holdridge, L.R., W.C. Grenke, W.H. Hatheway, T. Liang \& J.A. Tosi. 1971. Forest environments in tropical life zones: A pilot Study. Pergamon, Oxford, England.

Howden, H.F. \& V.G. Nealis. 1975. Effects of clearing in the tropical rain forest on the composition of the coprophagous scarab beetle fauna (Coleoptera). Biotropica 7: 77-83.

Jiménez-Ferbans, L. \& G. Amat-García. 2009. Sinopsis de los Passalidae (Coleoptera: Scarabaeoidea) del Caribe Colombiano. Caldasia 31: 155-173.

Klein, B.C. 1989. Effects of forest fragmentation on dung and carrion beetle communities in Central Amazonia. Ecology 70: 1715-1725.

Köppen, W. 1936. Das geographische System der Klimate. In W. Koeppen \& R. Geiger (eds.). Vol. 5, parte C. Handbuch der Klimatologie. Gebrueder Borntraeger, Berlin, Germany.

Krebs, C. 1988. Ecología: Estudio de la distribución y la abundancia. Oxford University, England.

Kremen, C. 1992. Assessing the indicator properties of species assemblages for areas monitoring. Ecological Appl. 2: 203-217.

Lenat, D.R. \& V.H. Resh. 2001.Taxonomy and stream ecology - The benefits of genus- and species-level identification. J. N. Am. Benthological Soc. 20: 287-298.
Magurran, A.E. 1988. Ecological diversity and its measurement. Princeton University, Princeton, Nueva Jersery, EE.UU.

Martínez, N.J., L.M. Cañas, J.L. Rangel, J.M. Barraza, J.M. Montes \& O.R. Blanco. 2010. Coleópteros coprófagos (Scarabaeidae: Scarabaeinae) en un fragmento de bosque seco tropical en el departamento del Atlántico, Colombia. Bol. Mus. Ento. U. Valle 11: 21-30.

Medina, C.A. \& A. Lopera. 2000. Clave ilustrada para la identificación de los géneros de escarabajos coprófagos (Coleoptera: Scarabaeinae) de Colombia. Caldasia 22: 299-315.

Morón, M.A. 1997. Inventarios faunísticos de los Coleoptera: Melolonthidae neotropicales con potencial como bioindicadores. G. Ital. Entomol. 8: 265-274.

Morón, M.A., B. Ratcliffe \& C. Deloya. 1997. Atlas de los escarabajos de México, Coleoptera: Lamellicornia, Vol. I Familia Melolonthidae. CONABIO-SME, México.

Morón, M.A. 2009. Chafers, rhinoceros and fruit beetles of the canopy in tropical forests. In: International Commission on Tropical Biology and Natural Resources. In K. Del Claro, P.S. Oliveira, V.R. Gray, A.A.A. Barbosa, A. Bonet, F. Rubio, F.J. Morales, L. Coelho, M.V. Sampaio, M.R. Morris, N. Ramirez, O. Marcal Junior, R.H. Ferraz, R.J. Marquis, S.C. Rodrigues \& U. Luttge (eds.). Encyclopedia of Life Support Systems (EOLSS), Developed under the Auspices of the UNESCO, Eolss, Oxford, Inglaterra (Consultado: 9 mayo 2011, http://www.eolss.net).

Neita, M.J.C., J. Orozco \& B. Ratcliffe. 2006. Escarabajos (Scarabaeidae: Pleurosticti) de la selva baja del bosque pluvial tropical "BP-T", Chocó, Colombia. Acta. Zool. Mex. 22: 1-36.

Nichols, E., T. Larsen, S. Spector, A.L. Davis, F. Escobar, M. Favila, K. Vulinec \& The Scarabaeinae Research Network. 2007. Global dung beetle response to tropical forest modification and fragmentation: a quantitative literature review and meta-analysis. Biol. Conserv. 137: 1-19.

Nichols, E., S. Spector, J. Louzada, T. Larsen, S. Amezquita, M.E. Favila \& The Scarabaeinae Research Network. 2008. Ecological functions and ecosystem services provided by Scarabaeinae dung beetles. Biol. Conserv. 141: 1461-1474.

Nichols, E., T.A. Gardner, C.A. Peres, S. Spector \& The Scarabaeinae Research Network. 2009. Co-declining mammals and dung beetles: an impeding ecological cascade. Oikos 118: 481-487.

Niemi, G.J., \& M.E. McDonald. 2004. Application of ecological indicators. Annu. Rev. Ecol. Syst. 35: 89-111.

Noriega, J.A., E. Realpe \& G. Fagua. 2007a. Diversidad de escarabajos coprófagos (Coleoptera: Scarabaeidae) 
en un bosque de galería con tres estadios de alteración. Universitas Scientiarum 1: 51-63.

Noriega, J.A., J.P. Botero, M. Viola \& G. Fagua. 2007b. Dinámica estacional de la estructura trófica de un ensamblaje de Coleoptera en la Amazonia Colombiana. Rev. Col. Entomol. 33: 157-164.

Noriega, J.A., A.M. Cubillos, C. Castañeda \& A.M. Sánchez. 2008. Actividad diaria de colonización del recurso alimenticio en un ensamblaje de escarabajos coprófagos (Coleoptera: Scarabaeidae) en la amazonía colombiana. Acta Biol. Col. 13: 75-86.

Noriega, J.A. \& G. Fagua. 2009. Monitoreo de escarabajos coprófagos (Coleoptera: Scarabaeidae) en la región neotropical, p. 165-188. In Técnicas de campo en ambientes tropicales. Manual para el monitoreo en ecosistemas acuáticos y artrópodos terrestres. In A. Acosta, G. Fagua \& A.M. Zapata (eds.). Pontificia Universidad Javeriana, Bogotá, Colombia.

Noss, R.F. 1990. Indicators for monitoring biodiversity: a hierarchical approach. Conserv. Biol. 4: 355-364.

Noss, R.F. 1999. Assessing and monitoring forest biodiversity: a suggested framework and indicators. Forest. Ecol. Manag. 115: 135-146.

Ocampo, F.C. 2006. Phylogenetic analysis of the scarab family Hybosoridae and monographic revision of the New World subfamily Anaidinae (Coleoptera: Scarabaeoidea). Bull. Univ. Nebraska St. Mus. 19: 3-177.

Otavo, S.E. 2010. Escarabajos (Coleoptera: Scarabaeoidea) como posibles elementos indicadores del estado de conservación de los bosques amazónicos: estudio de caso en el Parque Nacional Natural Amacayacu. Trabajo de grado, Ingeniería Forestal, Universidad Distrital Francisco José de Caldas, Bogotá, Colombia.

Pardo-Locarno, L.C., B. Ramírez-Pava, H. Villota, O. Villanueva \& W. Bahamon. 2011. Ensamblaje de escarabajos Melolonthidae (Coleoptera: Scarabaeoidea) asociados con pasturas en el departamento del Caquetá y su posible relación con la salubridad edáfica. Acta Agro. 60: 273-284.

Parques Nacionales Naturales. 2005. Plan de Manejo 20052009. Parque Nacional Natural Amacayacu. Parques Nacionales Naturales de Colombia, Ministerio de Medio Ambiente, Vivienda y Desarrollo Territorial, Bogotá, Colombia.

Pearce, J.L. \& L.A. Venier. 2006. The use of ground beetles (Coleoptera: Carabidae) and spiders (Araneae) as bioindicators of sustainable forest management: a review. Ecol. Indicators 6: 780-793.

Pearson, D.L. 1994. Selecting Indicator Taxa for the Quantitative Assessment of Biodiversity. Philos Trans R Soc London [Biol] 345: 75-79.

Pineda, E., C. Moreno, F. Escobar \& G. Halffter. 2005. Frog, bat, and dung beetle diversity in the cloud forest and coffee agroecosystems of Veracruz, México. Conserv. Biol. 19: 400-410.

Pinilla, M.C. 2004. Uso del paisaje en el sector sur del Parque Natural Nacional Amacayacu (AmazonasColombia). Cuadernos Desarrollo Rural 53: 133-156.

Pulido, L.A., R.A. Riveros, F. Gast \& P. Von Hildebrand. 2003. Escarabajos coprófagos (Coleoptera: Scarabaeidae: Scarabaeinae) del Parque Nacional Natural "Serranía de Chiribiquete", Caquetá, Colombia (Parte I). m3m-Monografías Tercer Milenio SEA Zaragoza. 3: $51-58$.

Ramírez, G.A. 1999. Ecología aplicada, diseño y análisis estadístico. Fundación Universitaria Jorge Tadeo Lozano, Bogotá, Colombia.

Ratcliffe, C.B. 2003. The Dynastinae Scarab Beatles of Costa Rica and Panama. University of Nebraska State Museum, Nebraska, EE.UU.

Restrepo, H., M.A. Morón, F. Vallejo, L. Pardo \& A. López. 2003. Catálogo de Coleoptera: Melolonthidae (Scarabaeidae: Pleurosticti) en Colombia. Folia Entomol. Mex. 42: 239-263.

Reyes, N.E. \& M.A. Morón. 2005. Fauna de Coleoptera Melolonthidae y Passalidae de Tzucacab y Conkal, Yucatán, México. Acta Zool. Mex. 21: 15-49.

Rudas, A. \& A.C. Prieto. 2005. Florura del Parque Nacional Natural Amacayacu. Amazonas, Colombia. Missouri Botanical Garden, Saint Louis, Missouri, EE.UU.

Ruiz, S.L., E. Sánchez, E. Tabares, A. Prieto, J.C. Arias, R. Gómez, D. Castellanos, P. García \& L. Rodríguez. 2007. Diversidad biológica y cultural del sur de la Amazonia colombiana-Diagnóstico. Corpoamazonia, Instituto Humboldt, Instituto Sinchi, UAESPNN, Bogotá, Colombia.

Scheffler, P.Y. 2005. Dung beetles (Coleoptera: Scarabaeidae) diversity and community structure across three disturban ceregimes in eastern Amazonia. J. Trop. Ecol. 21: 9-19.

Schulze, C.H., M. Waltert, P.J.A. Kessler, R. Pitopang, Shahabuddin, Veddeler, M.D. Mühlenberg, S.R. Gradstein, C. Leuschner, I. Steffan-Dewenter \& T. Tscharntke. 2004. Biodiversity indicator groups of tropical land-use systems: comparing plants, birds, and insects. Ecol. Appl. 14: 1321-1333.

Schuster, J.C. 1993. Passalidae: clave para géneros de Colombia. Bol. Mus. Ento. U. Valle 1: 55-61.

Schuster, J.C., E.B. Cano \& C. Cardona. 2000. Un método sencillo para priorizar la conservación de los bosques nubosos de Guatemala, usando Passalidae (Coleoptera) como organismos indicadores. Acta Zool. Mex. 80: 197-209.

Schuster, J.C. \& E. Cano. 2005. Clave para los géneros de los Passalidae americanos. (Consultado: 7 marzo 2011, http:/www.unl.edu/museum/research/ 
entomology/Guide/Scarabaeoidea/Passalidae/Passalidae-Key/Passalidaeclave.pdf).

Solís, A. 2007. Métodos y Técnicas de Recolecta para Coleópteros Scarabaeoideos. Instituto Nacional de Biodiversidad, Santo Domingo, Heredia, Costa Rica (Consultado: 7 marzo 2011, http://www.inbio.ac.cr/ papers/meto-col-scarabaeoidea/metoscar.pdf).

Solís, C., J.A. Noriega \& G. Herrera. 2011. Escarabajos coprófagos (Coleoptera: Scarabaeinae) en tres bosques secos del departamento del Atlántico-Colombia. Bol. Mus. Ento. U. Valle 12: 33-41.

Stork, N.E., T.J.B. Boyle, V. Dale, H. Eeley, B. Finegan, M. Lawes, N. Manokaran, R. Prabhu \& J. Soberon. 1997. Criteria and indicators for assessing the sustainability of forest management: conservation of biodiversity. CIFOR Working paper. CIFOR, Bogor, Indonesia.

Suárez, G. \& G. Amat-García.2007. Lista de especies de los escarabajos fruteros (Melolonthidae: Cetoniinae) de Colombia. Biota Col. 8: 69-76.

Triplehorn, C.A. \& N.F. Johnson. 2005. An introduction to the study of insects. Thomson Brooks/Cole. Belmont, California, EE.UU.

Uehara-Prado, M., J.D. Fernandes, A.D. Bello, G. Machado, A.J. Santos, F.Z. Vaz-de-Mello \& A.V.L. Freitas. 2009. Selecting terrestrial arthropods as indicators of small-scale disturbance: A first approach in the Brazilian Atlantic Forest. Biol. Conserv. 142: 1220-1228.

Vidaurre, T., L. Gonzales \& M. Ledezma. 2008. Escarabajos coprófagos (Scarabaeidae: Scarabaeinae) del palmar de las islas, Santa Cruz-Bolivia. Kempffiana 4: 3-20.

Villarreal, H., M. Álvarez, S. Córdoba, F. Escobar, G. Fagua, F. Gast, H. Mendoza, M. Ospina \& A.M. Umaña. 2006. Insectos, p. 151-183. In Manual de métodos para el desarrollo de inventarios de biodiversidad segunda edición. Programa de Inventarios de Biodiversidad. Instituto de Investigación de Recursos Biológicos Alexander Von Humboldt, Bogotá, Colombia.

Vitolo, A. 2004. Guía de la identificación de los escarabajos tigre (Coleoptera: Cicindelidae) de Colombia. Instituto de Investigación de Recursos Biológicos Alexander Von Humboldt, Bogotá, Colombia.

Yahner, H.R. 1988. Changes in wildlife communities near edges. Conserv. Biol. 2: 333-339.

Zambrano, H., M.E. Pardo \& L.G. Naranjo. 2007. Metodología para evaluar integridad ecológica en áreas protegidas del Sistema de Parques Nacionales de Colombia. Parques Nacionales Naturales, WWF Colombia, Instituto de Investigación en Recursos Biológicos Alexander Von Humboldt, Bogotá, Colombia. 